\title{
Resistive Plate Chambers working at high rate
}

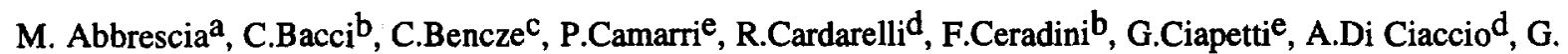

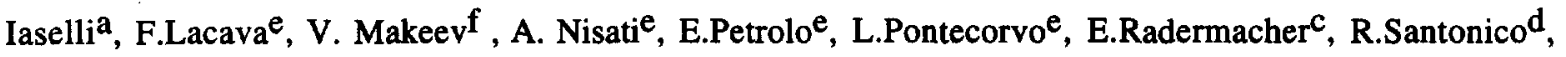

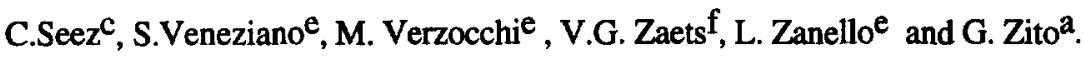

a Dipartimento di Fisica dell'Università di Bari and INFN - Sezione di Bari - Italy

b Dipartimento di Fisica della Terza Università di Roma and INFN - Sezione di Roma I - Italy

c CERN, Geneva, Switzerland

d Dipartimento di Fisica dell'Università di Roma "Tor Vergata" and INFN - Sezione di Roma II - Italy

e Dipartimento di Fisica dell'Università di Roma "La Sapienza" and INFN - Sezione di Roma I - Italy

f IHEP, Protvino, Russia.

presented by A. Di Ciaccio

Resistive Plate Chambers operating at low gas amplification have been tested in the RD5 experiment at CERN on a high intensity pion beam together with a $14 \mathrm{mCi} 60 \mathrm{CO}$ source to give an uniform photon background, simulating conditions close to those expected for an LHC muon detector.

\section{Introduction}

The Resistive Plate Chambers were used for a long time in low rate experiments at a gas gain around $10^{8}[1]$. In this operating mode the charge of the signals induced on the pick-up strips is around $100 \mathrm{pC}$ and limits the maximum rate at which the detector can be efficiently operated at values around $100 \mathrm{~Hz} / \mathrm{cm}^{2}$ [2].

The RPCs have been proposed [3] for the construction of large area $\mu$ trigger for the ATLAS and CMS detectors at LHC thanks to their spacetime resolution and low cost. Recent Montecarlo calculations [4] have shown that the secondary interactions of high energy particles with the detectors and accelerator elements gives a severe source of radiation background. An improvement in the RPCs rate capability was required to safely operate RPCs at LHC.

In a previous paper ${ }^{[5]}$ it has already been pointed out that a lower gas amplification $\left(10^{6}+\right.$ $10^{7}$ ) together with a frontend electronics with high amplification and bandwidth increases the rate capability of the detector. These ideas have been confirmed by several measurements [6].
In this paper we presents further results concerning RPC working at low gas gain. The chambers were simultaneously irradiated with high intensity pion beams and with a $14 \mathrm{mCi}{ }^{60} \mathrm{Co}$ source which insured an uniform photon background.

\section{Experimetal lay-out}

The RPCs tested are three single gap chambers, with double read-out as shown in fig. 1 . They are built with bakelite plate of volume resistivity about $0.5 \times 10^{11} \Omega \mathrm{cm}$, dimension $50 \times 50$ $\mathrm{cm}^{2}$ and thickness $2 \mathrm{~mm}$.

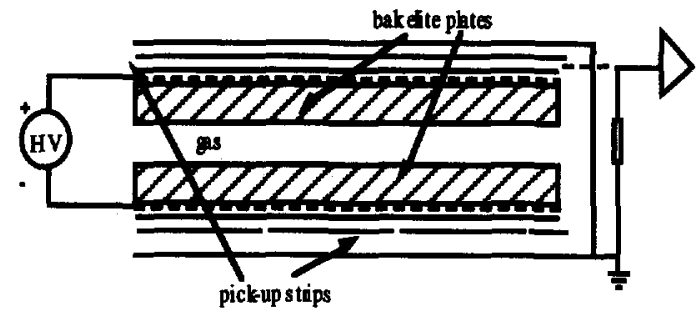

Figure 1. Double sided strip readout RPC. 
The signals are read out by 40 pick-up strips, $10 \mathrm{~mm}$ wide, with a $12 \mathrm{~mm}$ pitch. The strips are terminated at both ends with $50 \Omega$ resistors. The frontend electronics was conceived to read out the signals in the low gas amplification working mode and consists of a voltage amplifier of bandwidth 500 $\mathrm{MHz}$ and amplification about 400 for the first chamber and 100 for the others. Only twelve strips in the centre of the chamber were connected to the preamplifiers. The amplified signals were sent to the counting room through $50 \Omega$ RG58 coaxial cables connected to $100 \mathrm{MHz}$ discriminators with the threshold set at $40 \mathrm{mV}$ and the output signal shaped at $20 \mathrm{~ns}$. The coincidence between the OR of the strips and the signal of the RD5 beam scintillators was used to measure the detector efficiency. The time resolution was measured with $0.5 \mathrm{~ns}$ resolution TDC using the RD5 trigger scintillators as a common start and the signal from the strips as independent stop signals.

The chambers were placed immediately upstream of the RD5 experimental area. The RD5 set-up is situated on the $\mathrm{H} 2$ beam of the CERN SPS North Area and has been extensively described elsewhere [7]. A $14 \mathrm{mCi}{ }^{60} \mathrm{Co}$ source was placed in front of the set-up on aside of the beam line, at a distance of about $65 \mathrm{~cm}$ from the first chamber, as shown in fig.2. A set of scintillator counters were used as trigger system. The pion beam was defined by a coincidences of three scintillators $\mathrm{S} 1, \mathrm{~S} 5, \mathrm{~S} 4$ of dimension $15 \times 15,10 \times 15$ and $2 \times 2 \mathrm{~cm}^{2}$ respectively. The beam profile was measured with two multiwire chambers $\mathrm{U} 1$ and $\mathrm{U} 3$ with $2 \mathrm{~mm}$ wire spacing.

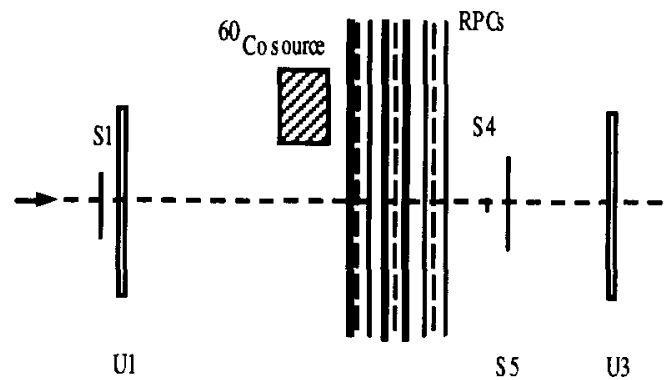

Figure 2. The RPCs experimental set-up.

\section{Experimental results}

The results presented here refer to RD5 runs taken in august 1994 with high intensity pion beams. They concerne only the chamber equipped with the higher frontend amplification. A gas mixture of butane and freon $\mathrm{CF} 3 \mathrm{Br}$ in various percentage (from $50 \%$ to $85 \%$ freon) was used.

The behavior of the RPC efficiency as a function of the high voltage is presented in fig. 3 , at different beam fluxes and for a gas mixure of $60 \%$ freon and $40 \%$ butane. The plateau efficiency is reached at higher voltages for higher rate since there is need to compensate for the reduction of the electric field in the gas due to the charge flowing through the resistive plates.

The rate capability of the chamber is shown in fig.4. The detector was filled with a mixture of $85 \%$ freon and $15 \%$ butane. Up to $1 \mathrm{KHz} / \mathrm{cm}^{2}$ the efficiency is about $97 \%$, then starts to decrease and at $10 \mathrm{KHz} / \mathrm{cm}^{2}$ it drops to $65 \%$. The quoted beam fluxes are average value measured with the multiwire chambers inside the beam spot and do not include the counting rate induced by Cobalt source. This rate at the beam spot position was about 150 $\mathrm{Hz} / \mathrm{cm}^{2}$, being the RPC sensitivity to low energy photons $\sim 10^{-2}[8]$.

In fig. 5 we present the time resolution as a function of the beam flux. The time resolution, defined as the RMS width of a gaussian fit to the time distribution, is $1.1 \mathrm{~ns}$ up to a beam flux of 1 $\mathrm{KHz} / \mathrm{cm}^{2}$, then starts to degrade and it is $2 \mathrm{~ns}$ at a beam flux of $10 \mathrm{KHz} / \mathrm{cm}^{2}$. The quoted resolutions include the substantial contribution of $0.9 \mathrm{~ns}$ due to the trigger scintillator jitter [2].

The distributions of the time delay of the chamber signals respect to the beam trigger scintillators versus the beam flux is presented in fig.6. The delay increase only of $1 \mathrm{~ns}$ when the flux ranges between 0.4 and $1 \mathrm{KHz} / \mathrm{cm}^{2}$, a considerable improvement respect to the high gas gain operation mode.

The total charge collected on the pick-up strips is shown in fig. 7 for a gas mixture $60 \%$ 

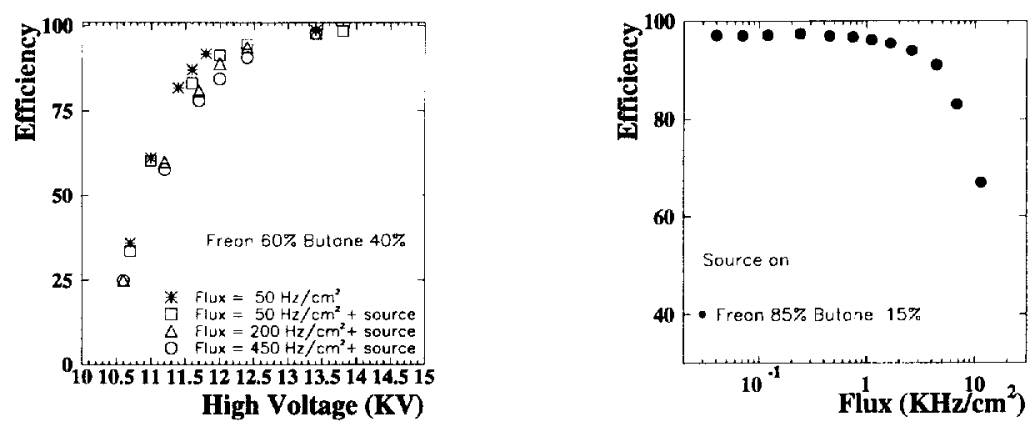

Figure 3: RPC efficiency versus HV

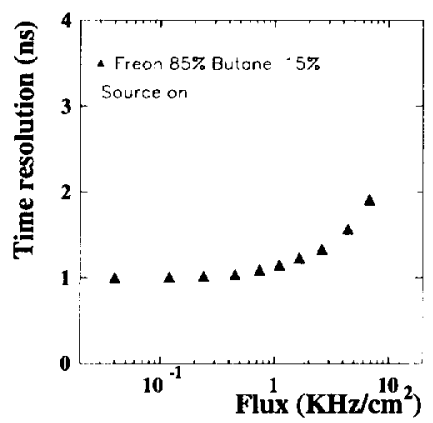

Figure 5: Time resolution versus flux

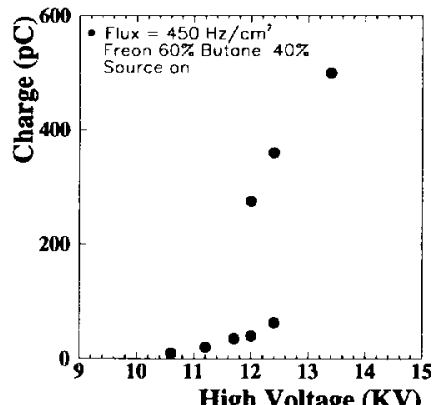

High Voltage (KV)
Figure 4: RPC efficiency versus flux

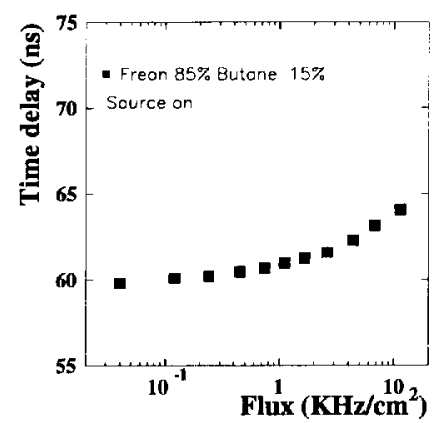

Figure 6: Time delay versus flux

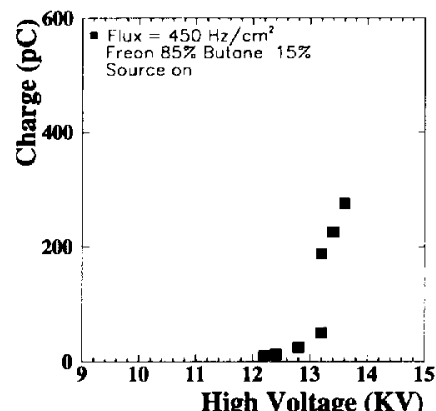

High Voltage (KV)

Figure 7: ADC total charge versus HV Figure 8: ADC total charge versus HV 
freon, $40 \%$ butane and in fig. 8 for $85 \%$ freon and $15 \%$ butane. The beam flux was $450 \mathrm{~Hz} / \mathrm{cm}^{2}$. A strip was contributing to the sum only if its charge was greater than three times the RMS of its pedestal. Taking into account the frontend amplification we have estimated the input charge. At the operating voltage giving fully efficiency it is about $1 \mathrm{pC}$ for the mixture $60 \%$ freon, $40 \%$ butane and $0.5 \mathrm{pC}$ for $85 \%$ freon and $15 \%$ butane.

\section{Conclusions}

We have tested RPCs chambers with double sided strip read-out operating at low gas gain, under a continuous and uniform irradiation of a photon source simulating LHC background conditions.

We have measured a rate capability $(97 \%$ efficiency at $1 \mathrm{KHz} / \mathrm{cm}^{2}$ ) and a time resolution (1.1ns up to a beam flux of $1 \mathrm{KHz} / \mathrm{cm}^{2}$ ) largely adequate to the LHC background conditions.

The total charge seen by the detector is about $0.5 \mathrm{pC}$ for a gas mixture $85 \%$ freon and $15 \%$ butane and becomes higher for higher freon content. We believe that this results show that RPCs are fully adequate for the muon trigger scheme of the ATLAS and CMS Technical proposals [9].

\section{References}

[1] R.Santonico and R.Cardarelli: Nucl.Instr.\& Meth 187 (1981) 377;R.Cardarelli et al.: Nucl.Instr.\& Meth A263 (1988) 20.

[2] M.Andlinger et al.(RD5 Collaboration): "Study of Resistive Plate Chambers for muon detection at hadron collider", NIM A 340 (1994) 370; M.Andlinger et al.: "Measurements of the efficiency and time resolution of double gap Resistive Plate Chambers ", Nucl.Instr.\& Meth A345 (1994) 474.

[3] F.Ceradini et al.: Proceeding of the Large Hadron Collider workshop, CERN/90-10, ECFA/90-133, eds. G.Jarlskog and D.Rein,
vol.III, pag 99; R. Santonico: Proceedings of the Large Hadron Collider Workshop, CERN/90-10,ECFA/90-133,edsG.Jarlskog nd D.Rein, vol.III, pag 838 .

[4] A.Ferrari: ATLAS Technical Note GEN-010.

[5] R.Cardarelli, A.Di Ciaccio and R.Santonico: Nucl.Instr.\& Meth A333 (1993) 399.

[6] R.Cardarelli ( RD5 Collaboration): Proceedings of the Workshop on Resistive Plate Chambers in particle Physics and Astrophysics,ed. S.Ratti, G.Ciapetti, and R. Santonico, Scientifica Acta 8(1993),pag.154;C.Bacci et al.:"Test of a Resistive Plate Chamber operating at low gas amplification at high intensity beams", Nucl.Instr.\& Meth A352 (1995) 552.

[7] M.Aalste et al.:"Measurement of hadron shower punchtrough in iron at the CERN SPS",Z. Phys. C 60(1993)1 10; H.Faissner et al: "Status Report of the RD5 experiment",CERN/DRDC/93-49.

[8] L. Acitelli et al.:"Study of the efficiency and time resolution of a RPC irradiated with photons and neutrons", Nota interna n 1039, Dip. di fisica dell' Universita di Roma, 15 luglio 1994.

[9] ATLAS,Technical Proposal,CERN/LHCC 194-43,LHCC/P2,15.12.1994;CMS, Technical Proposal, CERN/LHCC/94-38,LHCC/P1, 15.12.1994. 